\title{
Surface and Friction Behavior of a Silicone Surfactant Adsorbed on Model Textiles Substrates
}

\author{
Xiaomeng Liu, ${ }^{\dagger}$ Junlong Song, ${ }^{\dagger, \ddagger}$ Dong Wu, ${ }^{\S}$ Jan Genzer," Thomas Theyson, ${ }^{\perp}$ and \\ Orlando J. Rojas $*,, \#$
}

Department of Forest Biomaterials, North Carolina State University, Raleigh, North Carolina 27695-8005, Jiangsu Provincial Key Laboratory of Pulp and Paper Science and Technology, Nanjing Forestry University, Nanjing, Jiangsu Province 210037, People's Republic of China, Department of Chemistry, Duke University, Durham, North Carolina 27708, Department of Chemical \& Bimolecular Engineering, North Carolina State University, Raleigh, North Carolina 27695-7905, TensTech Inc., 424 Shrewsbury Lane, Matthews, North Carolina 2815, and Department of Forest Products Technology, Faculty of Chemistry and Materials Sciences, Aalto University, P.O. Box 16300, FI-00076 Aalto, Espoo, Finland

\begin{abstract}
This study reports on interactions of an amphiphilic block copolymer of polyalkylene oxide-modified poly(dimethylsiloxane) with thin films of polypropylene (PP), polyethylene terephthalate (PET), and nylon, as well as with reference hydrophilic silica surfaces. The dynamics of adsorption, adsorbed mass, and viscoelasticity of the adsorbed layer are quantified by using a quartz crystal microbalance, while boundary layer lubrication behaviors are studied by using lateral force microscopy. Driven by hydrophobic interactions, the silicone surfactant adsorbs following a Langmuir isotherm and forms strongly adsorbed layers on the polymer surfaces with an areal mass directly related to the hydrophobicity of the substrate. The self-assembled silicone surfactant molecules improve significantly wettability and lower friction. The results reported herein will broaden our understanding of lubrication phenomena in textile and fiber processing applications.
\end{abstract}

\section{Introduction}

Silicone-based surfactants comprising permethylated siloxane hydrophobic groups coupled to one or more polar groups, usually nonionic poly(ethylene oxide) (PEO) and poly(propylene oxide) (PPO), are commonly used in various industrial applications. ${ }^{1}$ Silicone-based surfactants have received increased attention due to their low surface tension, wetting properties, and the fact that they do not produce abrasion when in contact with human skin. Therefore, surface-active silicone-based molecules are used widely, for example, in the formulations of household and personal care products, wetting additives, polyurethane foams, coatings, etc. ${ }^{2-8}$ They have been investigated extensively with regard to their properties in solution, and their interfacial and wetting performance. ${ }^{1-23}$

Silicone surfactants are known for their suitability in applications involving either aqueous or nonaqueous media. This property is not common in surfactant chemistries where mixtures, for example, those involving short-chained alcohols, are likely to reduce molecular association. Surface and bulk characteristics of fabrics, such as softness, bounciness, tear strength, dry feel, wet feel, and hydrophilicity, can be enhanced significantly by surface finishes based on silicone surfactants. They are important components in the processing of textile fibers to control friction, wear, and other surface and interfacial functions. As compared to typical organic additives, siliconbased textile lubricants are reported to possess excellent performance and durability while being cost-effective. ${ }^{1}$

* To whom correspondence should be addressed. Tel.: (919) 5137494. Fax: (919) 515-6302. E-mail: ojrojas@ ncsu.edu.

Department of Forest Biomaterials, North Carolina State University.

Nanjing Forestry University.

$\S$ Duke University.

"Department of Chemical \& Bimolecular Engineering, North Carolina State University.

${ }^{\perp}$ TensTech Inc.

\# Aalto University.
Studies on the adsorption behavior of silicone surfactants on solid surfaces, especially those relevant to textile processing, are very limited. ${ }^{24-26}$ The spreading properties of silicone surfactants with different structures in contact with hydrophobic surfaces have been investigated. ${ }^{26}$ It was found that the presence of water vapor was necessary to attain superspreading capabilities, while such an effect appeared not to be critically affected by the molecular geometry of the investigated (trisiloxane) surfactant.

Wang et al. measured the interaction force profile of three silicone surfactants adsorbed on n-octadecyltrichlorosilane substrates in the presence of ethanol using atomic force microscopy. The researchers found that the surfactant adsorbed and self-assembled onto the hydrophobic surfaces driven by the hydrophobic effect. ${ }^{24,25}$ As expected, the hydrodynamic thickness of the adsorbed surfactant layer increased with increasing chain length of its hydrophilic moiety. An interesting property of adsorbed silicone-based surfactants is their stability against alcohols and other solvents. It was reported that alcohols provide interfacial stabilization in aqueous medium via steric repulsions at ethanol concentrations up to $80 \%$. This is in contrast to nonionic surfactants based on ethylene and polypropylene oxide, which lose surface activity at approximately $40 \%$ ethanol concentration. ${ }^{24,25}$

The present study addresses the adsorption of a commercial silicone surfactant and the influence of substrate hydrophobicity on the surface properties. The main determinations include the dynamics of adsorption, the mass, viscoelasticity, and stability of the adsorbed layers, and the wetting and boundary layer lubrication behaviors. Adsorption experiments are performed by using quartz crystal microgravimetry (QCM), and lateral forces are measured via atomic force microscopy (AFM). The main objective is to reveal the critical features displayed by molecular assemblies of the silicone surfactant upon adsorption onto textile-relevant surfaces, including hydrophobic polypro- 


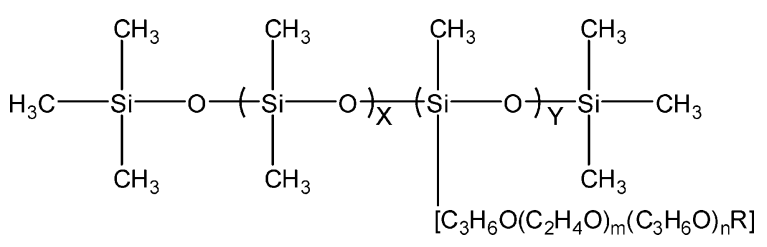

Figure 1. Chemical formula of the main component of the silicone surfactant used in this investigation. ${ }^{28}$

pylene (PP), polyethylene terephthalate (PET), and nylon, and to compare the results with reference hydrophilic silica substrates.

\section{Experimental Section}

Materials. All experiments were performed with deionized water from an ion-exchange system (Pureflow, Inc.) followed by a Milli-Q Gradient unit (resistivity $>18 \mathrm{M} \Omega$ ). Silica wafers (cut into $1.5 \times 1.5 \mathrm{~cm}^{2}$ pieces) were obtained from Wafer World Inc., FL. Gold- and silica-coated quartz sensors used in the experiments with quartz crystal microgravimetry (QCM) were acquired from Q-Sense (Västra Frölunda, Sweden).

Medium density polypropylene, PP (syndiotactic PP with $M_{\mathrm{n}}$ and $M_{\mathrm{w}}$ of 54 and $127 \mathrm{kDa}$, respectively), and nylon 6 (3 mm particle size, $T_{\mathrm{g}}=62.5^{\circ} \mathrm{C}$ ) were purchased from Sigma-Aldrich. Polyethylene terephthalate (PET) was provided by Goulston Inc. (Monroe, NC).

The mixture with silicone surfactant under trade name D190 Fluid (Dow Corning MI, USA) was used without further purification. It consisted of dimethyl, methyl (propyl(poly(EO)(PO))acetate) siloxane as the main component (see Figure 1) and poly(ethylene oxide propylene oxide) monoallyl ether acetate with small amounts of polyether polyol acetate. The dimethicone copolyol compounds are usually produced in equilibrium reactions, which result in complex mixtures of homologous oligomeric products that nevertheless impart a synergistic effect in terms of their surface activities. ${ }^{4,27}$ Before application the mixture, thereafter referred to as silicone surfactant, it was diluted with milli-Q water until reaching given concentrations, with no electrolytes added. These aqueous solutions were used in surface tension, adsorption, and lateral force measurements.

Methods. QCM sensors were treated first with Piranha solution (70:30, $\left.\mathrm{H}_{2} \mathrm{SO}_{4}: \mathrm{H}_{2} \mathrm{O}_{2}(30 \%)\right)$ for $20 \mathrm{~min}$ followed by $10 \mathrm{~min} \mathrm{UV/ozone} \mathrm{treatment}\left(28 \mathrm{~mW} / \mathrm{cm}^{2}\right.$ at $\left.254 \mathrm{~nm}\right)$ to remove any organic contaminants, immediately before spin-coating them with the respective polymer. Thin films of PP, PET, and nylon were deposited by spin-coating on clean QCM gold sensors; details about their preparation can be found in ref 29. Samples for measurement of water contact angle, before and after treatment with silicone surfactant solutions, were obtained from flat silica wafers that were subjected to the same cleaning and spin-coating procedures as those used in the case of QCM sensors.

Adsorption of Silicone Surfactant. The rate of silicone surfactant adsorption and key characteristics of the adsorbed layer, described further below, were assessed by a quartz crystal microbalance (Q-Sense model E4, Gothenburg, Sweden). The changes of resonant frequency $f$ and energy dissipation $D$ of the polymer-coated QCM sensors were measured simultaneously by switching on and off the applied voltage. The principles of the QCM technique have been addressed in detail elsewhere. ${ }^{30-32}$ Briefly, changes in the mass and viscoelasticity of the adsorbed layer produced a shift in the QCM frequency $(\Delta f)$ and energy dissipation $(\Delta D)$ that were used to determine these properties. ${ }^{33}$ The frequency and dissipation values for the third, fifth, and

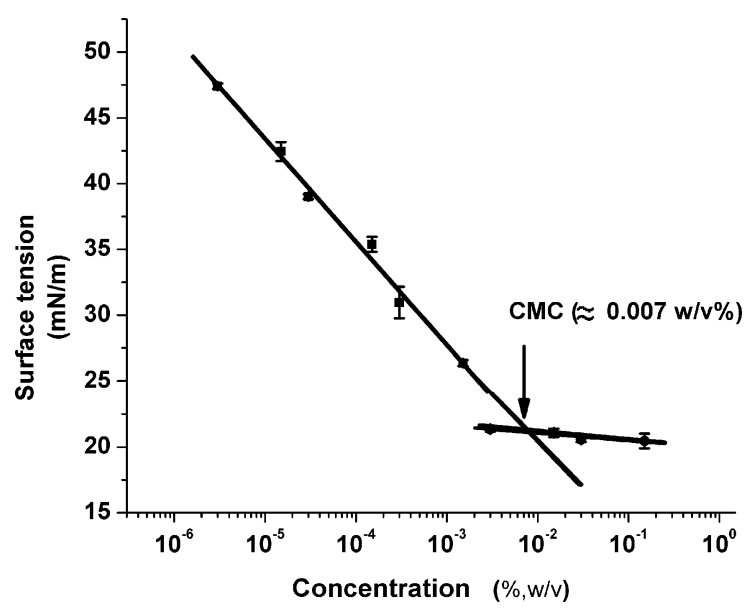

Figure 2. Surface tension isotherm of the silicone-based surfactant used in this investigation $\left(25^{\circ} \mathrm{C}\right)$.

seventh overtones were recorded, and from the data the dynamics of adsorption was determined. $\Delta D$ was measured by recording the exponential decay of oscillation (frequency and amplitude dampening), which allowed quantification of the energy dissipated and stored during one period of oscillation, $E_{\text {dissipated }}$ and $E_{\text {stored }}$, respectively (see eq 1 ):

$$
\Delta D=\frac{E_{\text {dissipated }}}{2 \pi E_{\text {stored }}}
$$

Silica- and polymer-coated sensors were mounted in the QCM module, and silicone surfactant aqueous solutions of given concentrations were injected continuously. In the preparation of the solution, the silicone surfactant was diluted with milli-Q water; no background electrolyte was used. The typical procedure used in adsorption experiments consisted of the following sequential steps: (1) Milli-Q water was injected through the QCM system, and the frequency shift was monitored to obtain a baseline. (2) Once a stable frequency signal was observed, it was set to zero, and surfactant solution with a given concentration (ranging from 0.00003 to $0.3 \mathrm{w} / \mathrm{v} \%$, i.e., below and above the CMC; see Figure 2) was injected. (3) The shift of QCM frequency and energy dissipation were then monitored until plateau values were reached. (4) A large volume of milli-Q water was injected to remove surfactant in the bulk solution as well as any molecules loosely bound to the surface. As a result of this rinsing step, new frequency and dissipation values, $f$ and $D$, respectively, were reached. These values indicated the effective changes produced by the adsorbing surfactant molecules, that is, in the absence of contributions from the bulk solution (density and viscosity) because the baseline and the plateau signal obtained after rinsing were both registered under the same aqueous medium. Shifts in $f$ and $D$ were monitored as a function of time for all polymer surfaces tested at $25^{\circ} \mathrm{C}$.

The viscoelasticity and thickness of the adsorbed film were extracted by fitting the QCM data to a Voigt viscoelastic model. ${ }^{34,35}$ The shifts in dissipation were $<5 \%$ of the respective frequency shift. Additionally, little differences among the normalized overtone frequencies and dissipations were registered. Therefore, the simpler Sauerbrey equation was found suitable to determine the adsorbed mass. The temperature was found to be critical in the adsorption experiments because it affected the density of the fluid flowing through the QCM module; therefore, it was held constant at $25 \pm 0.02{ }^{\circ} \mathrm{C}$ in all experiments. 
Surface Tension. The surface tension of silicone surfactant solutions was determined at $22{ }^{\circ} \mathrm{C}$ by means of a Cahn balance (Thermo Material Characterization, Madison, WI) equipped with a Pt-Ir Willhelmy plate. The minimum surface tension and the critical micelle concentration (CMC) were determined.

Contact Angle Measurement. The water contact angles (WCA) on the different surfaces were measured with a Phoenix 300 system (SEO Corp., Korea) by delivering a $4 \mu \mathrm{L}$ volume drop from a needle connected to a syringe pump. The images of the sessile drop were analyzed with respect to their width and height to yield the contact angle and drop volume by using the "Image J" software (National Institutes of Health, U.S.). The contact angles of the polymer substrates were assessed both before and after silicone surfactant adsorption from $0.03 \mathrm{w} / \mathrm{v} \%$ aqueous solutions, that is, above the critical micelle concentration (CMC) and after the substrates were gently blown dry with nitrogen gas in a laminar flow cabinet. The averages of at least three contact angles for each condition and substrate are reported here. The difference in water contact angles measured before and after strong sonication in water (during $15 \mathrm{~min}$ at $42 \mathrm{kHz}$ $\pm 6 \%$ ) was also determined to check the stability or durability of the adsorbed surfactant layers.

AFM Lateral Forces. An atomic force microscope, AFM (MFP3D, Asylum Research Group), was employed to measure the friction forces. The experiments were conducted on silica wafers coated with the respective polymer (PP, nylon, or PET). Scans were performed separately in air, water, and silicone surfactant solution after adsorption of the silicone surfactant from 0.03 wt $\%$ aqueous solution concentrations $(4.3 \times \mathrm{CMC})$, followed by rinsing with water and drying. At this concentration, a plateau in surfactant adsorption and coverage is expected, as will be shown later. All of the measurements were performed in contact mode using a scan size and rate of $200 \mathrm{~nm}$ and 2.0 $\mathrm{Hz}$, respectively, with a constant normal force of $\sim 32.6 \mathrm{nN}$, which was determined by using the method of Behary et.al. ${ }^{36}$ The cantilever used was CSC17 (Mikromasch AFM probe) with tips comprising Al backside coated Si. AFM lateral forces were used in friction determinations. To decouple the contributions from the chemistry of the surface and that of the topography, forward and backward scans (trace and retrace) were performed in the same area of the sample. Lubrication due to the adsorbed boundary layer was accounted for by the extent of AFM tip twisting; effects arising from differences in surface topography or roughness were considered to ensure that the net friction was determined. ${ }^{36}$ System normal force sensitivity and cantilever calibration were performed before every measurement, and the normal force was kept the same in all runs. No attempt was made to calibrate the torsional forces of the cantilevers, although a number of methods have been used. ${ }^{37-40}$ Instead, a simple approach based upon the normalization of friction coefficients with internal standards was carried out, as has been suggested elsewhere. $^{41}$

\section{Results and Discussion}

The main component of the silicone surfactant used in this study was a surface-active block copolymer of polyalkylene oxide-modified poly(dimethylsiloxane). Because most poly(dimethylsiloxanes) are nonpolar, their aqueous solutions exhibit very low surface tensions. In fact, a sharp drop of surface tension with concentration was observed (see Figure 2), from $\sim 47$ $\mathrm{mN} / \mathrm{m}$ for $3 \times 10^{-6} \mathrm{w} / \mathrm{v} \%$, the lowest concentration used, to $\sim 21 \mathrm{mN} / \mathrm{m}$ for $3 \times 10^{-3} \mathrm{w} / \mathrm{v} \%$ concentration, slightly below the critical micelle concentration (CMC of $7 \times 10^{-3} \mathrm{w} / \mathrm{v} \%$ ). The surface tension above the CMC decreased slightly with concentration. Surface activity, surface tension reduction, and CMC were comparable to those reported for dimethicone polyols ${ }^{27}$ and other nonionic surfactants but not as low as reported in the literature for pure silicone surfactants. This is mainly due to the characteristic molecular composition of the surfactant formulation used and the presence of other components in the mixture.

The surface excess $\Gamma$ at the air/liquid interface was obtained by the Gibbs adsorption isotherm:

$$
\Gamma=-\frac{1}{R T}\left(\frac{\partial \gamma}{\partial \ln c}\right)_{T}
$$

where $\gamma$ is the surface tension (in the present case measured at constant temperature of $297.2 \mathrm{~K}$ ), $R$ is the universal gas constant, and $c$ is the silicone surfactant concentration. At maximum polymer packing, the calculated surface excess was 0.83 molecules $/ \mathrm{nm}^{2}\left(0.124 \mathrm{~nm}^{2} /\right.$ molecule $)$. Similarly to the case of the air/liquid interface, adsorption is expected to occur via the nonpolar groups interacting with the low energy solid surfaces driven by the hydrophobic effect. It is also expected that the surfactant molecules self-assemble onto the surface and form boundary layers that are effective in reducing wear and friction.

Dynamics of Adsorption. Adsorption from solution was determined by using the QCM, as explained in the Methods. Before every experiment, water was injected continuously in the QCM's sampling loop until a stable baseline was obtained. Silicone surfactant solution was then injected at a low volumetric flow rate of $0.1 \mathrm{~mL} / \mathrm{min}$. As a result of the injection of the silicone surfactant solution, sharp changes in frequency and dissipation were observed. The plateau QCM frequency $f$ and dissipation $D$ after silicone surfactant adsorption were recorded before and after rinsing with water (see Figure 3 for the third overtone $f$ and $D$ ). The dynamics of the adsorption process was evaluated from frequency shift profiles. The net change in QCM frequency and dissipation, which are related to the adsorbed mass and the viscoelasticity of the adsorbed layer, are shown in Figure $3 \mathrm{a}$ and $\mathrm{b}$, respectively. Profiles similar to those observed for silica surfaces were obtained when adsorption occurred on the hydrophobic polymeric substrates. In all cases, the observed QCM profiles were consistent with molecular adsorption on PP, PET, and nylon surfaces (polymer-coated QCM sensors). Typically, the adsorption process reached a plateau $\sim 10$ min after injection, as judged by stable frequency and dissipation signals. Data acquisition under these stable conditions continued for more than $50 \mathrm{~min}$ to ensure that adsorption equilibrium was achieved. This equilibrium condition was then challenged by rinsing with water. As a result, sharp changes in frequency and dissipation were observed. The increase in $f$ indicated that loosely bound molecules were removed, and therefore only a thinner, less dissipative adsorbed layer remained on the surface.

At $\sim 15-20 \mathrm{~min}$ after rinsing, higher frequency and lower dissipation plateau values were reached, as compared to the values measured prior rinsing. On the basis of the difference in surfactant chemical potential, the adsorbed mass obtained from the shift in frequency was classified as reversible and irreversible for the plateau values before and after rinsing, respectively. The shift in frequency before rinsing was larger than that after rinsing. Furthermore, at concentrations below the $\mathrm{CMC}$, both adsorption values became larger as the surfactant concentration was increased; however, a level-off signal, indicative of negligible changes in the adsorbed mass, was observed at solution concentrations above the CMC.

Changes in energy dissipation depicted in Figure 3b suggest that before rinsing the surfactant adsorbed as a loose structure, 

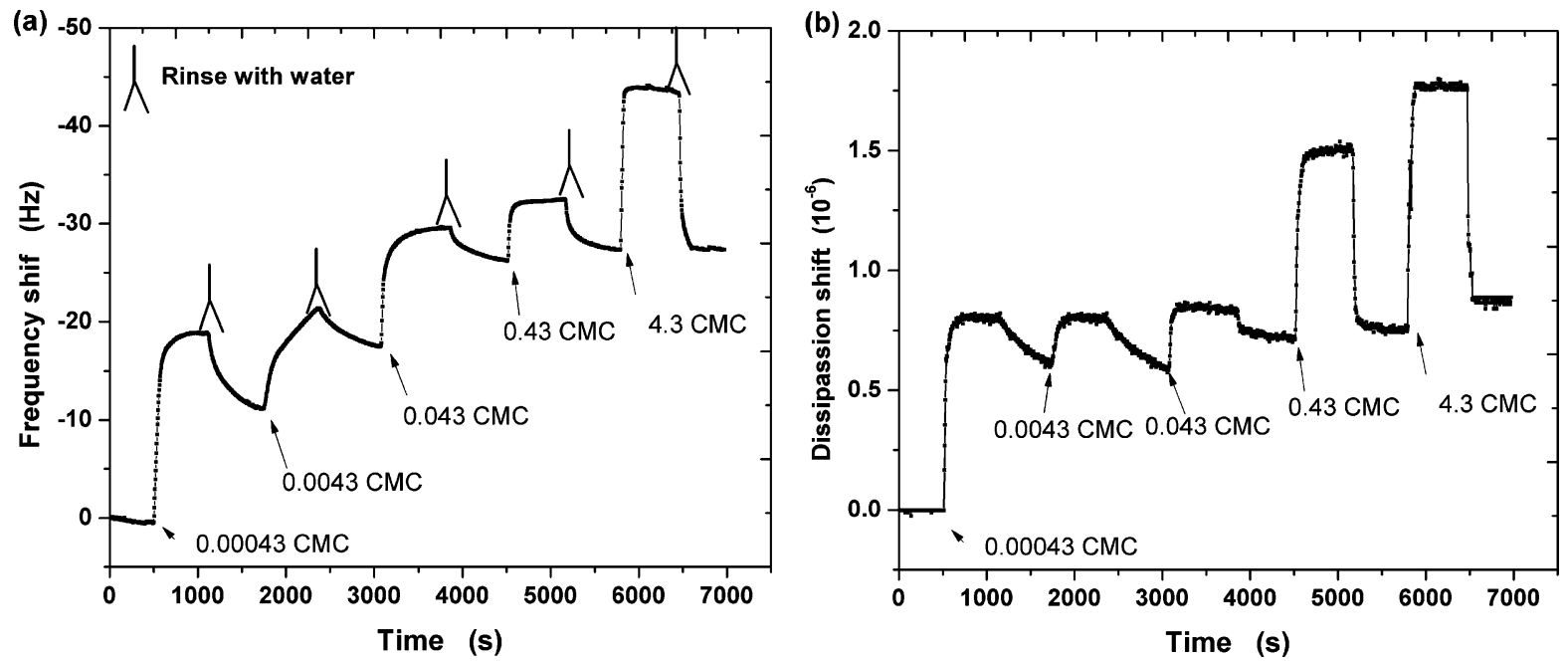

Figure 3. Mean values of third overtone QCM frequency (a) and energy dissipation (b) as a function of time upon injection of silicone surfactant solution on silica surfaces at various aqueous solution concentrations (from $3 \times 10^{-6}$ to $3 \times 10^{-2} \mathrm{w} / \mathrm{v} \%$ ) indicated by the respective arrows for concentrations expressed as multiple units of the CMC. The experiments were conducted in open (continuous) flow configuration and with an injection rate of $0.1 \mathrm{~mL} / \mathrm{min}$. The dips observed in the profiles after adsorption plateau were produced after rinsing the system with water. Behaviors similar to that observed for silica were obtained for PP, PET, and nylon surfaces (data not shown).
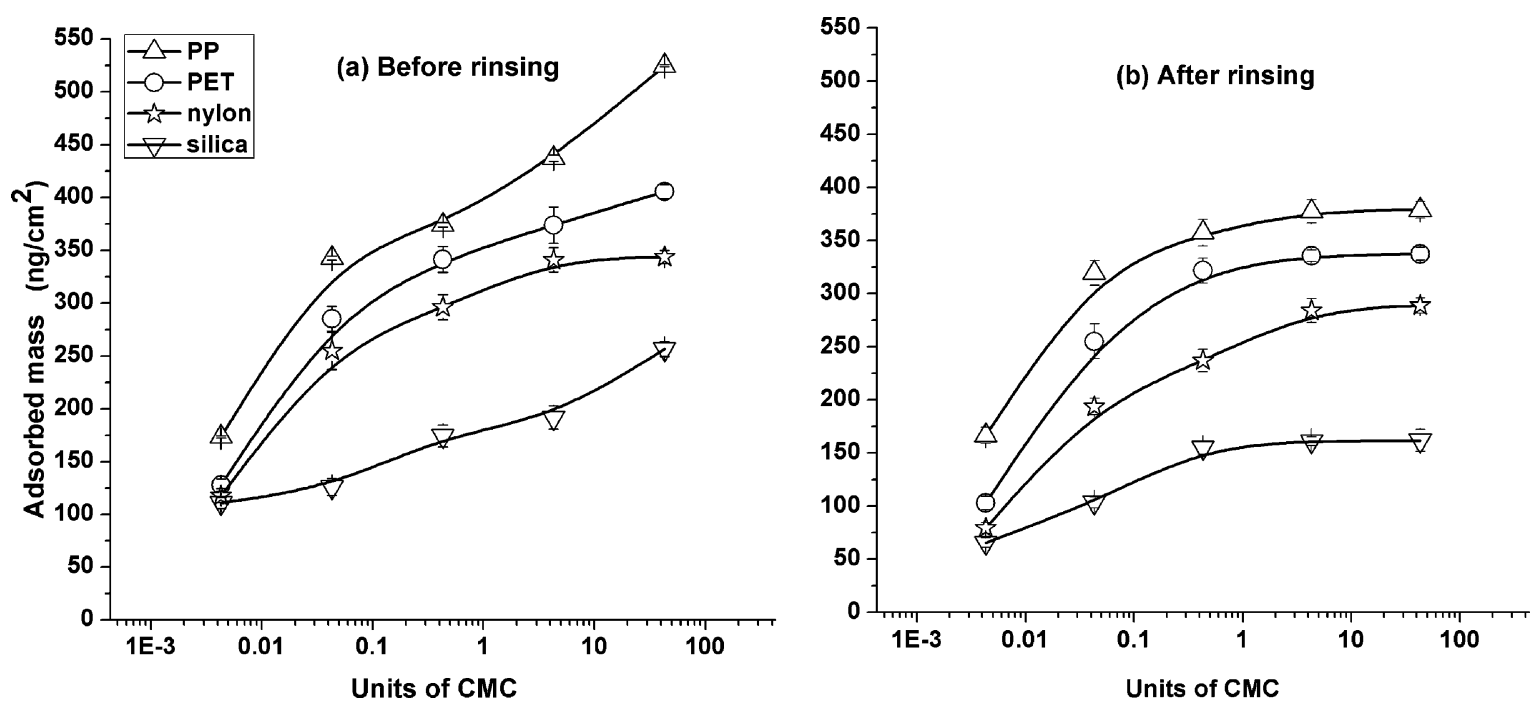

Figure 4. Sauerbrey adsorbed mass for silicone-based surfactants adsorbing on PP, PET, nylon, and silica before rinsing (reversible adsorption, a) and after rinsing (irreversible adsorption, b) with water. The experimental standard deviation for all data collected is shown as error bars for each condition. The lines are meant to guide the eyes.

while the layer that remained on the surface after rinsing showed the characteristic energy dissipation of a relatively thin and rigid layer with an absolute dissipation shift of no more than $1 \times$ $10^{-6}$ units. Supporting evidence for the formation of a very thin adsorbed layer was obtained after observing overlapping values of the normalized QCM frequency and dissipation for the various overtones (data not shown).

Adsorption Isotherms. The adsorbed masses on the substrates considered, that is, thin films of PP, PET, and nylon as well as silica, were calculated from the Sauerbrey equation using the values of QCM frequency shifts for the given surfactant concentration, before and after rinsing. The plateau values before and after rinsing were used to plot the isotherms seen in Figure 4. The changes in the reversible adsorption, before rinsing (cf., Figure 4a), depended linearly on the surfactant bulk concentration. Rinsing with water produced some limited reduction in the amount of silicone surfactant sensed at the interface. The adsorbed mass measured after rinsing indicated some removal of loosely bound surfactant molecules, but a large number of segments remained adsorbed, likely due to an energy barrier that prevented their desorption.

It is worth noting that the QCM signal is sensitive to coupled water, especially for highly hydrated or solvated molecules, as has been shown for adsorbed nonionic triblock copolymers. ${ }^{42}$ In the case of the data corresponding to irreversibly adsorbed mass, that is, that obtained after rinsing (cf., Figure 4b), plateau adsorption was reached at a concentration slightly above the CMC. It can be argued that this state corresponds to an adsorbed monolayer, which could be modeled by a Langmuir-type isotherm. The surface density $\Gamma$ of adsorbed molecules was thus expressed as a function of solute concentration in the aqueous phase, $c$, via the Langmuir isotherm (see eq 3), which was used to fit the experimental data (see fitted lines in Figure 4b).

$$
\Gamma\left(X, C_{i}\right)=\frac{Q_{0} b C_{i}}{1+b C_{i}}
$$

On the basis of the frequency shifts and the corresponding adsorbed mass, it can be concluded that the extent of silicone 
Table 1. Water Contact Angles of the Surfaces before and after Adsorption of Silicone Surfactant

\begin{tabular}{lcc}
\hline & \multicolumn{2}{c}{ WCA $(\mathrm{deg})$} \\
\cline { 2 - 3 } & bare surfaces & surfaces after surfactant adsorption \\
\hline PP & $103 \pm 1$ & $13 \pm 0.5$ \\
PET & $69 \pm 1$ & $19 \pm 1$ \\
nylon & $61 \pm 1$ & $16 \pm 1$ \\
silica & $1 \pm 0.5$ & $8 \pm 1$
\end{tabular}

adsorption increased with the substrate hydrophobicity; it was largest on PP, followed by PET, nylon, and silica.

The water contact angles (WCA) of untreated PP, PET, nylon, and silica surfaces were $\sim 102^{\circ}, \sim 69^{\circ}, \sim 61^{\circ}$, and $1^{\circ}$, respectively. A larger hydrophobic driving force, which is related to the initial WCA, led to more effective adsorption of the silicone molecules. Additionally, a stronger ability to overcome the steric barriers imposed by adsorbed chains was present in the more hydrophobic substrates that otherwise would have prevented further adsorption.

Adsorption on PP, PET, and nylon could be expected to occur in the form of a surfactant monolayer, at least at concentrations below the CMC. The fact that the adsorbed amount levels off at values of concentration close to the CMC supports this hypothesis. However, the presence of adsorbed association structures, that is, adsorbed micelles, patchy bilayers, cannot be ruled out. In such situations, the aggregated surface structures may form with extensive chain interpenetration.

In studies related to adsorption phenomena, Yoon and Ravishankar ${ }^{43-45}$ found that the decay length of hydrophobic forces depended on the hydrophobicity of the surface: below a "transition" regime for surfaces with WCA $\theta_{\mathrm{a}} \approx 90^{\circ}$, the hydrophobic forces were short-ranged, and above this contact angle they were described as long-ranged. The water contact angles of bare PP surfaces were the highest, $102^{\circ}$, while those for PET, nylon, and silica were $69^{\circ}, 61^{\circ}$, and $1^{\circ}$, respectively. The highest WCA for PP is indicative of inherently higher hydrophobicity of PP due to its $-\mathrm{CH}_{2}-$ and $-\mathrm{CH}_{3}$ groups. In contrast to PP, nylon and PET, which contain carboxyl and carbonyl groups, are more polar. It is hypothesized that the larger contribution to the irreversible adsorption arises from the hydrophobic forces, while van der Waals interactions are predominant in the reversible adsorption, as shown by others. ${ }^{24,25}$ In summary, silicone-based surfactants adsorbed to a larger extent onto the more hydrophobic surfaces are likely driven by the hydrophobic effect.

Integrity of the Adsorbed Surfactant Layers. Water contact angles were measured on the surfaces after silicone surfactant adsorption from aqueous solution concentrations above the CMC $(4.3 \times \mathrm{CMC})$; see Table 1 . For each substrate, adsorption was allowed overnight followed by water rinsing and drying with gentle nitrogen jet. After adsorption of the silicone-based surfactant, a sharp reduction in water contact angle was observed for PP, PET, and nylon. This was in contrast to the case of the highly hydrophilic silica where the contact angle was increased to $8^{\circ}$.

The stability of the adsorbed silicone molecules on different polymer surfaces was further tested by exposing the different samples to high energy sonication. In these experiments, after adsorption and rinsing, the polymer surfaces were subjected to sonication in water for 5, 10, and $15 \mathrm{~min}$. After drying with a gentle nitrogen jet, the WCA was measured on each substrate. An increase in WCA, as compared to that measured for similar samples that were not subject to sonication, could be attributed to the release or depletion of the adsorbed layer. However, the results from these experiments revealed that only a very limited change in WCA was observed in the case of PP, PET, and nylon (the maximum WCA increase was between $3^{\circ}$ and $7^{\circ}$ ), regardless of the sonication time (see Figure 5 as an illustration of the case of PP surfaces). Therefore, the adsorbed layers of silicone surfactant on these surfaces were found to be very stable.

Friction Forces. The friction forces of AFM tip sliding against layers of silicone surfactant adsorbed on PP, PET, nylon, and silica were measured in air, water, and in the presence of the silicone surfactant solution. A static friction force on the tip was expected to occur when the tip started to move on the given surface. This static friction force was evident in the force profile obtained in air during the forward and the backward scanning. The difference between the backward and forward lateral force signals is related to twice the friction force. ${ }^{31}$ The friction coefficients were determined by dividing the friction force by the normal applied force, the latter being calculated by using the contact force curve profile for an applied constant normal force of $32.6 \mathrm{nN}$ (see the Supporting Information), and normalized by dividing each value by the magnitude of the coefficient of friction calculated for silica in air. Multiple measurements of the friction force were performed, and the average data were recorded on different days and using different cantilevers. Comparison of the absolute friction coefficients reported here with those from other instruments can be only exercised with caution. However, the relative magnitude of the coefficients of friction determined for the different surfaces reported is reliable due to the internal consistency and calibration used in such determination; furthermore, experimental uncertainties are expected to be significantly smaller than those that result from measurements based on absolute deflection determinations.

Figure 6 shows the relative friction forces on adsorbed layers of silicone surfactant preadsorbed on PP (a), PET (b), nylon (c), and silica (d) and measured in air, water, and silicone surfactant solution. From Figure 5a, it can be observed that the friction force in air was always the highest, because there was no interfacial fluid present. When the surfaces were immersed in water, the friction force was reduced sharply, confirming the lubricating effect of water. With addition of the silicone surfactant, the friction force was reduced significantly, as compared to that measured in water. However, when the tip was sliding on a surface with adsorbed surfactant molecules, the high force from the static friction disappeared, and it was replaced by an oscillating force curve. As expected, the amplitude of force curve in the solution was much smaller than that in air. Therefore, friction was reduced to a large extent by the presence of a boundary lubricant layer consisting of the adsorbed silicone surfactant molecules.

Friction force trends similar to those observed for PP were observed in the case of PET and nylon (see Figure $6 a-c$ ). After the surfaces were immersed in water, the friction forces reduced sharply. With addition of the silicone surfactant solution, the friction force was reduced further. Interestingly, in the case of silica surfaces (Figure 6d), such an effect was not observed. This can be explained by the adsorption data discussed earlier, which indicated a smaller adsorption on silica.

Figure $7 \mathrm{a}$ shows the relationship between adsorption amount (in $\mathrm{ng} / \mathrm{cm}^{2}$ ) and the normalized friction coefficient (see the Supporting Information) for silicone surfactant adsorbed from 4.3 $\times$ CMC surfactant solutions. It can be observed that the friction coefficient decreased with the adsorbed mass of silicone surfactant.

Figure $7 \mathrm{~b}$ shows the mean, normalized values of the friction coefficient for different surfaces measured in air, water, and silicone lubricant. The normalization was carried out relative to values for silica measured in air. The data reported were obtained after averaging at least three values obtained for the different samples. 


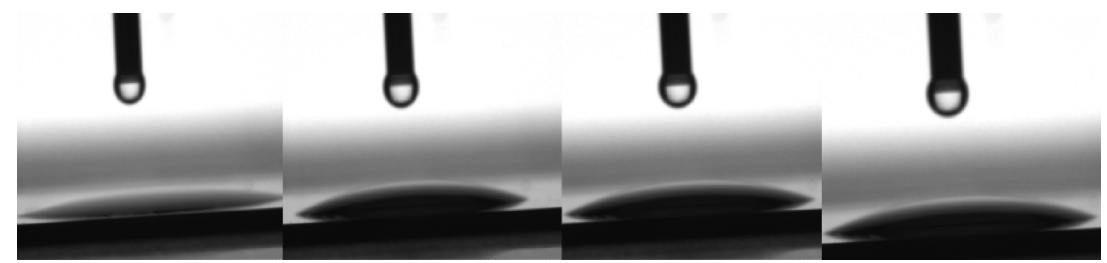

Figure 5. Water droplets on modified PP surfaces after 0, 5, 10, and 15 min sonication treatment.

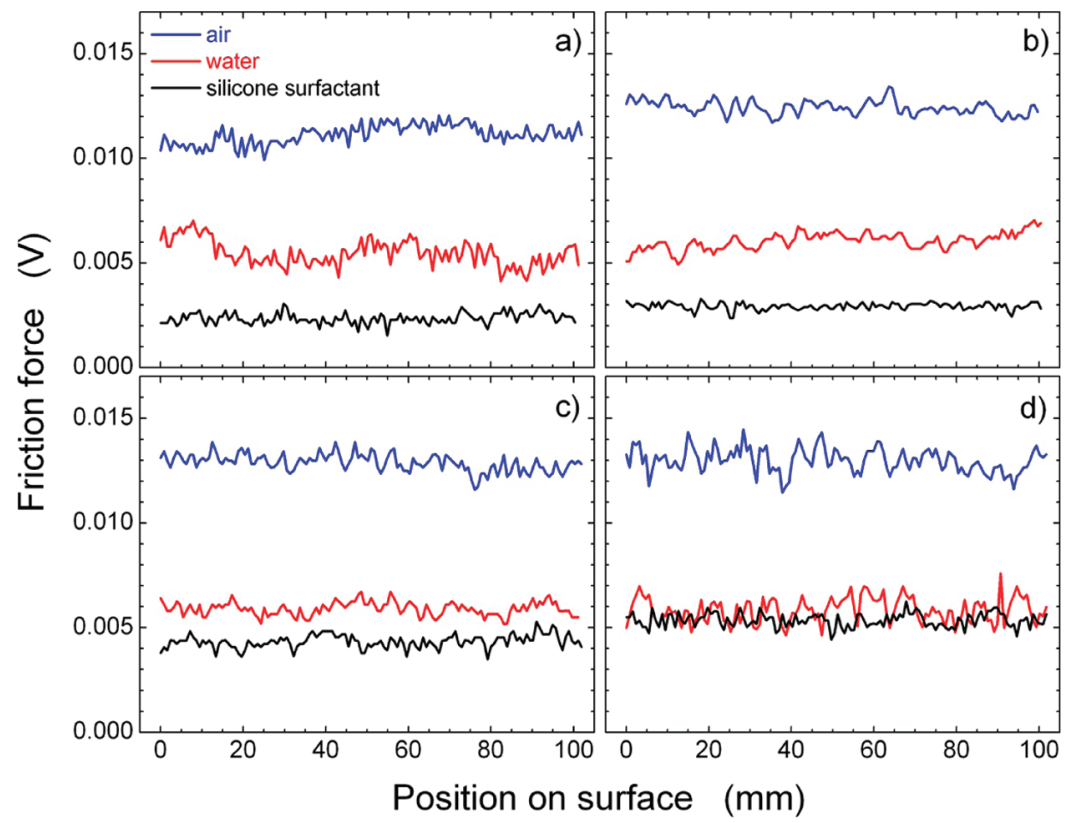

Figure 6. AFM friction force signal as a function of the position of the AFM tip scanned on a layer of silicone-based surfactant adsorbed on PP (a), PET (b), nylon (c), and silica (d). The lateral force measurements were performed in three different media, air, water, and silicone surfactant solution $(4.3 \times \mathrm{CMC}$ or $0.03 \mathrm{w} / \mathrm{v} \%)$.
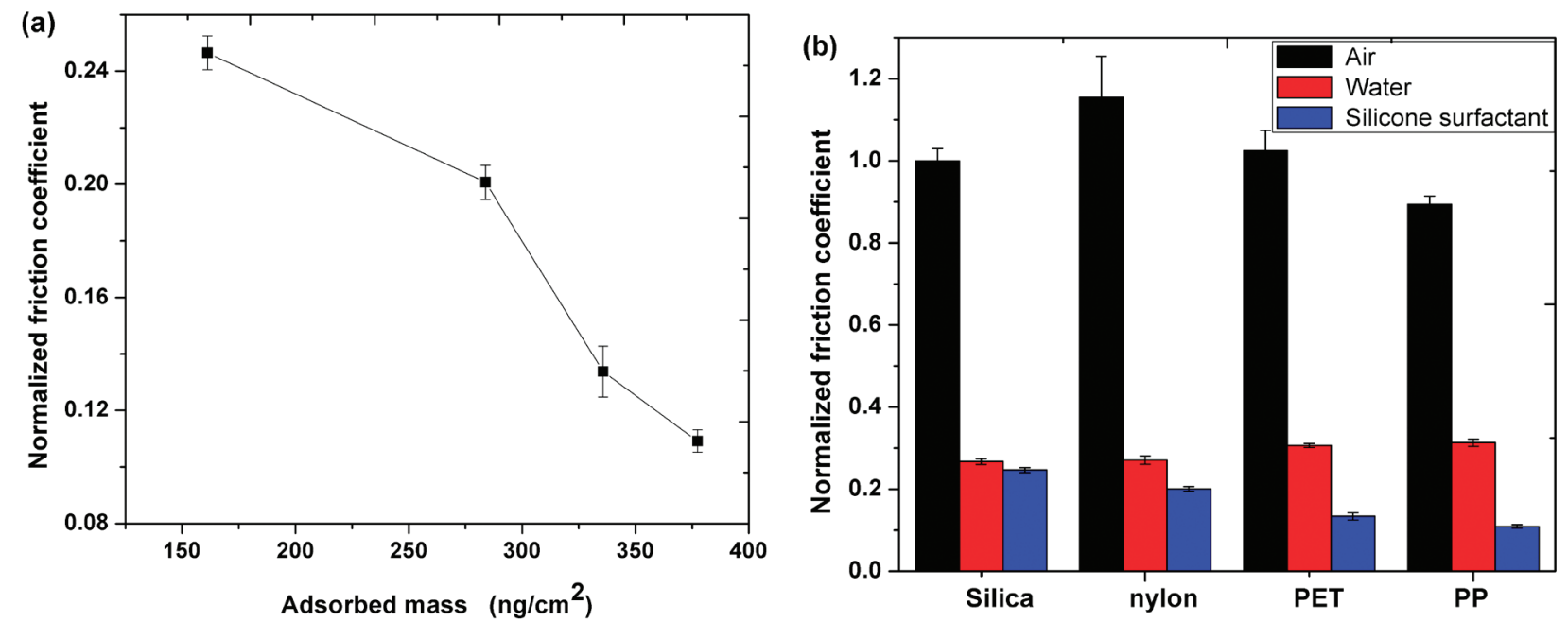

Figure 7. (a) Relationship between adsorption amount $\left(\mathrm{ng} / \mathrm{cm}^{2}\right)$ and friction coefficient (normalized with respect to silica surfaces measured in air) for silicone surfactant adsorbed from $4.3 \times$ CMC solution on PP, PET, nylon, and silica surfaces. (b) Normalized friction coefficient for different surfaces measured with a bare tip in air, water, and silicone surfactant solution.

The largest normalized coefficients of friction were measured in air as was described before for friction forces. The magnitude of the friction coefficients for the different surfaces is expected to be related to their respective chemical nature and surface energy. As noted before, when the surfaces were immersed in water, the coefficient of friction reduced sharply. The friction coefficients measured in water increased slightly with the hydrophobicity of the substrates. The differences in the coefficients of friction may thus be attributed to a combination of factors including the difference in surface free energy and roughness. The probe, in this case a standard commercial silicon tip that may be oxidized under the ambient conditions, was polar and thus was expected to interact more strongly with the more polar silica surface due to higher adhesion. Furthermore, as shown in an extensive body of literature, the stronger adhesion correlates with increased friction forces. ${ }^{37}$

However, a distinctive observation in the present case was the higher friction coefficient for the more nonpolar surfaces. This fact may be explained by other contributions such as 
surface roughness, that is, 1.8, 1.1, 0.8, and $0.1 \mathrm{~nm}$ for PP, PET, nylon, and silica, respectively, as reported in earlier work. ${ }^{29}$

The friction coefficients were reduced sharply after addition of the silicone lubricant followed by rinsing with water. Interestingly, the trend in the coefficient of friction as a function of substrate hydrophobicity was reversed; that is, the more hydrophobic substrates yielded lower coefficients of friction. The underlying effect that would explain these trends is related to the minimization of the interfacial energy by self-assembly of the adsorbing silicone surfactant, which was more pronounced in the case of the more hydrophobic substrates.

It is hypothesized that the hydrophobic segments of the silicone surfactant adsorbed on PP, PET, and nylon surfaces, while the hydrophilic segments acted as buoys in the liquid medium. In the case of silica surfaces, the hydrophilic segments of the silicone lubricant were adsorbed to the surface, and the hydrophobic segments probably associated with other similar segments. In aqueous medium, hydrophilic-hydrophilic interactions are weak and shear-sensitive, for example, to rinsing with water. The adsorption amount was reduced after rinsing, as shown in the discussion on adsorption; that is, the irreversible adsorption of silicone surfactants on silica was lower than that on PP, PET, and nylon surfaces.

Surfactant molecules adsorbed on the surfaces and formed a boundary layer that altered the chemistry of the surface. The adsorbed layer thickness and mass as well as its structure are expected to be crucial in lubrication phenomena. In the present cases, the silicone-based surfactant provided better lubrication performance on the more hydrophobic polymer substrates. This fact is supported by common knowledge in that these types of surfactants perform ideally on hydrophobic fibers, especially on PP.

Physisorption as a dominant effect in our experimental observations is relevant to textile processing. It is envisioned that typical lubricant molecules or finishes are not intended to remain adsorbed on the surface of the fibers. A strong interaction would lead to lubricant layers that would interfere with successive processes, including surface finishing and dyeing. While the evidence presented here indicates that a very thin layer remains adsorbed irreversibly on the surface of PP, PET, and nylon, other factors, such as shear stress and the application of harsher rinsing steps, with solutions of different composition and/or ionic strength, could lead to important variations in the surface chemistry of the treated substrates.

\section{Conclusions}

Silicone surfactants have attracted attention in textile processing due to their excellent surface activity, high performance, and durable hydrophilicity. In this study, the surface activity of a commercial silicone surfactant and its adsorption behavior on substrates with different hydrophobicities were investigated by using quartz crystal microgravimetry and scanning probe microscopy. The relationship between surfactant adsorbed amount and lubrication performance was revealed. The following conclusions can be drawn from the results reported in this study: (1) Silicone surfactants possess high surface activity, with minimum surface tensions of the order of $\sim 20 \mathrm{mN} / \mathrm{m}$. (2) The hydrophobic moiety of the silicone surfactant interacts with the hydrophobic substrates through hydrophobic forces; high affinity isotherms are observed. (3) The silicone surfactant adsorption affinity was found to be related directly to the hydrophobicity of the substrate. (4) The water contact angle of textile-relevant surfaces was improved to a large extent due to the spreading and wetting effects of the adsorbed silicone surfactants. (5) The obtained self-assembled surfactant layers reduced friction and are expected to be effective to prevent wear in the conditions of boundary lubrication. Finally, the adsorbed silicone layers were found to be robust and durable.

\section{Acknowledgment}

This project was supported by the Nonwovens Cooperative Research Center Project number 07-98.

Supporting Information Available: AFM spring constant, lateral sensitivity, and normal force calibration results. This material is available free of charge via the Internet at http:// pubs.acs.org.

\section{Literature Cited}

(1) Hill, R. M. Silicone surfactants - new developments. Curr. Opin. Colloid Interface Sci. 2002, 7, 255.

(2) Hill, R. M. NetLibrary Inc. Silicone Surfactants; Marcel Dekker: New York, 1999; p 360.

(3) Christiano, S. P.; Fey, K. C. Silicone antifoam performance enhancement by nonionic surfactants in potato medium. J. Ind. Microbiol. Biotechnol. 2003, 30, 13.

(4) O'Lenick, A. J. Silicone emulsions and surfactants. J. Surfactants Deterg. 2000, 3, 387.

(5) Grundke, K.; Michel, S.; Knispel, G.; Grundler, A. Wettability of silicone and polyether impression materials: Characterization by surface tension and contact angle measurements. Colloids Surf. 2008, 317, 598.

(6) Jha, B. K.; Christiano, S. P.; Shah, D. O. Silicone antifoam performance: Correlation with spreading and surfactant monolayer packing. Langmuir 2000, 16, 9947

(7) Lin, L. H.; Wang, C. C.; Chen, C. W.; Chen, K. M. Water-repellency and antibacterial activities of plasma-treated cleavable silicone surfactants on nylon fabrics. Surf. Coat. Technol. 2006, 201, 674.

(8) Somasundaran, P.; Mehta, S. C.; Purohit, P. Silicone emulsions. Adv. Colloid Interface Sci. 2006, 128, 103.

(9) La Torre, C.; Bhushan, B.; Yang, J. Z.; Torgerson, P. M. Nanotribological effects of silicone type, silicone deposition level, and surfactant type on human hair using atomic force microscopy. J. Cosmet. Sci. 2006, $57,37$.

(10) Gentle, T. E.; Snow, S. A. Adsorption of small silicone polyether surfactants at the air/water interface. Langmuir 1995, 11, 2905.

(11) Kaneko, M.; Matsuzawa, K.; Uddin, M. H.; Lopez-Quintela, M. A.; Kunieda, H. Effect of hydrophobic chain length of amphiphilic silicone oil (copolymer) on the nonionic surfactant-layer curvature. J. Phys. Chem. B 2004, 108, 12736.

(12) Kunieda, H.; Uddin, M. H.; Horii, M.; Furukawa, H.; Harashima, A. Effect of hydrophilic- and hydrophobic-chain lengths on the phase behavior of A-B-type silicone surfactants in water. J. Phys. Chem. B 2001, 105,5419 .

(13) Kuo, P. L.; Hou, S. S.; Teng, C. K.; Liang, W. J. Function and performance of silicone copolymer (VI). Synthesis and novel solution behavior of water-soluble polysiloxanes with different hydrophiles. Colloid Polym. Sci. 2001, 279, 286.

(14) Li, X.; Washenberger, R. M.; Scriven, L. E.; Davis, H. T.; Hill, R. M. Phase behavior and microstructure of water/trisiloxane E-6 and E-10 polyoxyethylene surfactant/silicone oil systems. Langmuir 1999, 15, 2278.

(15) Lin, L. H.; Chen, K. M. Surface activity and water repellency properties of cleavable-modified silicone surfactants. Colloids Surf. 2006, $275,99$.

(16) Neumann, B.; Vincent, B.; Krustev, R.; Muller, H. J. Stability of various silicone oil/water emulsion films as a function of surfactant and salt concentration. Langmuir 2004, 20, 4336.

(17) Pandya, K. P.; Lad, K. N.; Bahadur, P. Silicone surfactants in aqueous solution 0.1. Surface activity and micellar behaviour. Tenside, Surfactants, Deterg. 1996, 33, 374.

(18) Silas, J. A.; Kaler, E. W.; Hill, R. M. Effect of didodecyldimethylammonium bromide on the phase behavior of nonionic surfactant-silicone oil microemulsions. Langmuir 2001, 17, 4534.

(19) Soni, S. S.; Sastry, N. V.; Aswal, V. K.; Goyal, P. S. Micellar structure of silicone surfactants in water from surface activity, SANS and viscosity studies. J. Phys. Chem. B 2002, 106, 2606.

(20) Soni, S. S.; Sastry, N. V.; George, J.; Bohidar, H. B. Dynamic light scattering and viscosity studies on the association behavior of silicone surfactants in aqueous solutions. J. Phys. Chem. B 2003, 107, 5382. 
(21) Soni, S. S.; Sastry, N. V.; Joshi, J. V.; Seth, E.; Goyal, P. S. Study on the effects of nonelectrolyte additives on the phase, thermodynamics, and structural changes in micelles of silicone surfactants in aqueous solutions from surface activity, small angle neutron scattering, and viscosity measurements. Langmuir 2003, 19, 6668.

(22) Uddin, M. H.; Rodriguez, C.; Watanabe, K.; Lopez-Quintela, A.; Kato, T.; Furukawa, H.; Harashima, A.; Kunieda, H. Phase behavior and formation of reverse cubic phase based emulsion in water/poly(oxyethylene) poly(dimethylsiloxane) surfactants/silicone oil systems. Langmuir 2001, 17, 5169.

(23) Yan, Y.; Hoffmann, H.; Drechsler, M.; Talmon, Y.; Makarsky, E. Influence of hydrocarbon surfactant on the aggregation behavior of silicone surfactant: Observation of intermediate structures in the vesicle-micelle transition. J. Phys. Chem. B 2006, 110, 5621.

(24) Wang, A. F.; Jiang, L. P.; Mao, G. Z.; Liu, Y. H. Direct force measurement of comb silicone surfactants in alcoholic media by atomic force microscopy. J. Colloid Interface Sci. 2001, 242, 337.

(25) Wang, A. F.; Jiang, L. P.; Mao, G. Z.; Liu, Y. H. Direct force measurement of silicone- and hydrocarbon-based ABA triblock surfactants in alcoholic media by atomic force microscopy. J. Colloid Interface Sci. 2002, 256, 331

(26) Zhu, S.; Miller, W. G.; Scriven, L. E.; Davis, H. T. Superspreading of water-silicone surfactant on hydrophobic surfaces. Colloids Surf. 1994, $90,63$.

(27) Anthony, J. O. Silicone emulsions and durfactants. J. Surfactants Deterg. 2000, 3, 387.

(28) Dow Corning 190 Fluid, product data sheet.

(29) Song, J. L.; Liang, J.; Liu, X. M.; Krause, W. E.; Hinestroza, J. P.; Rojas, O. J. Development and characterization of thin polymer films relevant to fiber processing. Thin Solid Films 2009, 517, 4348.

(30) Sauerbrey, G. The use of quartz oscillators for weighing thin layers and for microweighing. Z. Angew. Phys. 1959, 155, 206.

(31) Edvardsson, M.; Rodahl, M.; Kasemo, B.; Höök, F. A dualfrequency QCM-D setup operating at elevated oscillation amplitudes. Anal. Chem. 2005, 77, 4918.

(32) Rodahl, M.; Kasemo, B. Frequency and dissipation-factor responses to localized liquid deposits on a QCM electrode. Sens. Actuators, B $\mathbf{1 9 9 6}$ $37,111$.

(33) Reimhult, E.; Larsson, C.; Kasemo, B.; Hook, F. Simultaneous surface plasmon resonance and quartz crystal microbalance with dissipation monitoring measurements of biomolecular adsorption events involving structural transformations and variations in coupled water. Anal. Chem. 2004, 76,7211 .
(34) Larsson, C.; Rodahl, M.; Hoöök, F. Characterization of DNA immobilization and subsequent hybridization on a $2 \mathrm{D}$ arrangement of streptavidin on a biotin-modified lipid bilayer supported on $\mathrm{SiO}_{2}$. Anal. Chem. 2003, 75, 5080.

(35) Voinova, M. V.; Rodahl, M.; Jonson, M.; Kasemo, B. Viscoelastic acoustic response of layered polymer films at fluid-solid interfaces: continuum mechanics approach. Phys. Scr. 1999, 59, 391.

(36) Behary, N.; Ghenaim, A.; Elachari, A.; Caze, C. Tribological analysis of glass fibers using atomic force microscopy (AFM)/lateral force microscopy (LFM). J. Appl. Polym. Sci. 2000, 75, 1013.

(37) Leggett, G. J.; Brewer, N. J.; Chong, K. C. Friction force microscopy: towards quantitative analysis of molecular organisation with nanometre spatial resolution. Phys. Chem. Chem. Phys. 2005, 7, 1107.

(38) Masalska, A.; Kolanek, K.; Woszczyna, M.; Zawierucha, P.; Ritz, Y.; Zschech, E. Calibration of Atomic Force Microscope for Nanoscale Friction Measurements. IEEE 2007, 1, 4244-1313.

(39) Bhushan, B. Handbook of Micro/Nanotribology; CRC Press: Boca Raton, FL, 1995.

(40) Fotiadis, D.; Scheuring, S.; Muller, S. A.; Engel, A.; Muller, D. J. Micron 2002, 33, 385.

(41) Barlow, I.; Sun, S.; Leggett, G. J.; Turner, M. Synthesis, monolayer formation, characterization, and nanometer-scale photolithographic patterning of conjugated oligomers bearing terminal thioacetates. Langmuir 2010, 26,4449 .

(42) Liu, X.; Wu, D.; Turgman-Cohen, S.; Genzer, J.; Theyson, T. W.; Rojas, O. J. Adsorption of a nonionic symmetric triblock copolymer on surfaces with different hydrophobicity. Langmuir 2010, 26, 9565.

(43) Yoon, R. H.; Ravishankar, S. A. Application of extended DLVO theory. 3. Effect of octanol on the long-range hydrophobic forces between dodecylamine-coated mica surfaces. J. Colloid Interface Sci. 1994, 166, 215.

(44) Yoon, R. H.; Ravishankar, S. A. Long-range hydrophobic forces between mica surfaces in alkaline dodecylammonium chloride solutions. J. Colloid Interface Sci. 1996, 179, 403.

(45) Yoon, R. H.; Ravishankar, S. A. Long-range hydrophobic forces between mica surfaces in dodecylammonium chloride solutions in the presence of dodecanol. J. Colloid Interface Sci. 1996, 179, 391.

Received for review June 3, 2010 Revised manuscript received July 31, 2010 Accepted August 4, 2010

IE1012213 